\title{
Seguridad Ciudadana en un Municipio Limeño: Análisis de los Resultados del Plan Local, 2017-2018
}

\section{Citizen Security in a Lima Municipality: Analysis of the Results of the Local Plan 2017-2018}

Iris Katherine Gonzales Figueroa

https://orcid.org/0000-0003-4335-192X

Miguel Bardales Cárdenas

https://orcid.org/0000-0002-1067-9550

Universidad Cesar Vallejo, Perú

Autor para correspondencia: mbardales2909@hotmail.com; mbardales@ucv.edu.pe; kgonzalesfigueroa@gmail.com; igonzales@ucv.edu.pe

Fecha de recepción: 23 de febrero del 2020 - Fecha de aceptación: 03 de julio del 2020

\section{Resumen}

El artículo tuvo como objetivo general comprobar los resultados alcanzados en la ejecución del plan local de seguridad ciudadana, en un municipio limeño, 2017-2018. El tipo de investigación fue participativa, enfoque cualitativo y un diseño de estudio de casos. Se recogieron las entrevistas de un comisario policial de distrito, de un experto en temas de asuntos policiales, de un empresario víctima de hechos delictivos y de un ex miembro dirigencial del Comité de Seguridad ciudadana del Distrito. Los principales hallazgos encontrados fueron: falta de compromiso $y$ responsabilidad de los representantes del Comité Local de Seguridad Ciudadana, alto índice de inseguridad en la zona, robos y asaltos que afectan los negocios, falta de efectivos policiales y serenos, alta rotación de comisarios, demora en la capacidad de respuesta de la policía ante la denuncia de un hecho delictivo e inmigración descontrolada. Las principales conclusiones fueron: nivel de eficacia de los planes de seguridad ciudadana solo alcanzaron un nivel valorativo medio de $(58.34 \%)$, inadecuado sistema de control del plan local de seguridad ciudadana e incumplimiento de las categorías apriorísticas contrastadas.

Palabras Claves: desarrollo humano; democracia; necesidades básicas; seguridad del Estado; seguridad internacional

\begin{abstract}
The article had the general objective of verifying the results achieved in the execution of the local plan of citizen security, in a municipality of Lima, 2017-2018. The type of research was participatory, qualitative approach and a case study design. Interviews were collected from a district police commissioner, an expert on police matters, a businessman who was the victim of criminal acts, and a former leadership member of the District's Citizen Security Committee. The main findings found were: lack of commitment and responsibility of the representatives of the Local Committee for Citizen Security, high rate of insecurity in the area, robberies and assaults that affect businesses, lack of police and serene personnel, high turnover of police officers, delay
\end{abstract}


in the capacity of the police to respond to the complaint of a criminal act and uncontrolled immigration, and as main conclusions we have: level of effectiveness of citizen security plans only reached an average value level of (58.34\%), inadequate control system of the local plan for citizen security and non-compliance with the contrasted a priori categories.

Key words: human development; democracy; basic needs; state security; international security

\section{Introducción}

Según Soto (2018) sostiene que un mundo globalizado la amenaza del terrorismo se ha vuelto cada vez inmediata y cercana, Alke (2018) manifiesta que la seguridad ciudadana es por lo general un tema dominado por militares e institutos especializados y García (2018) expresa que la frontera tiene una connotación importante; manifestando que a partir del 11 se septiembre del año 2001, cuando ocurrieron los luctuosos actos terroristas contra los dos centros comerciales de New York y el Departamento de Defensa; los Estados Unidos que hasta ese entonces practicaba otro concepto en materia de seguridad nacional, cambia su postura y empieza una gran reorganización de todas sus instituciones que tenían que ver con la seguridad nacional. El expresidente estadounidense George W. Bush en sus dos periodos de gobierno (2001-2009), inicia una estrategia denominada "guerra preventiva" y crea la Agencia Nacional de Seguridad (NSA) que unifica todas las agencias de investigación e inteligencia, con la finalidad de unificar el comando bajo una sola autoridad. Esta política de alguna manera no sufrió cambios significativos con su sucesor Obama (2009-2017). Europa no estuvo exenta de atentados terroristas, como lo sucedido en España el 11 de marzo del 2004 (11M) y el 8 de agosto del 2017; Francia el 13 de noviembre del 2017 y Londres en el año 2017. En un mundo globalizado, como el actual, es el terrorismo una de las amenazas principales, que influye en las percepciones de los ciudadanos en cuanto a las condiciones se seguridad.

El Perú no ha estado exento del incremento de la delincuencia y criminalidad, hasta el mes de abril del año 2018, se estima que el $25.5 \%$ de los ciudadanos peruanos fueron víctimas de algún hecho delictivo, lo cual representa que uno de cada cuatro peruanos fue víctima de algún acto delincuencial. Respecto a la Prov. De Lima la tasa de victimización fue del $29.1 \%$. y en cuanto a la percepción de inseguridad ciudadana, a abril del año 2018, esta fue del 84.9\%, y en el caso de la Prov. De Lima, la tasa fue del 90.3\%. Si bien la percepción de inseguridad ciudadana tiene un componente de tipo emocional y de momento; por el hecho de ver y conocer mediante los medios de comunicación y escuchar de boca de sus familiares y/o amigos hechos de carácter delictuoso, determinan la creencia que es posible ser víctima de algún tipo de delito, ( Ministerio del Interior , 2018, pág. 7).

La justificación de la presente investigación se sustenta, en una concepción teórica, es decir parte de los postulados de la investigación cualitativa, buscando respuestas bajo conceptos apriorísticos y paradigmas vigentes (integridad, multisectorialidad, rigurosidad, sostenibilidad e intensidad), vinculados a la seguridad ciudadana, y como estos responden al tratamiento de un fenómeno eminentemente social. Su justificación metodológica, se sustenta en que pone a prueba el diseño estudio de caso, para entender y comprender un fenómeno directamente desde sus actores presenciales. Consideraciones que no son recogidas o conocidas si se investigaran de manera cuantitativa. La justificación practica responde porque busca soluciones a problemas de la ciudad como los robos y asaltos a las personas y viviendas, pandillaje, asesinatos, violaciones,

Esta obra se comparte bajo la licencia Creative Common Atribución-No Comercial 4.0 International (CC BY-NC 4.0) Revista de la Universidad Internacional del Ecuador. URL: https://www.uide.edu.ec/ 
feminicidios, que son los principales delitos que afectan a los ciudadanos de este distrito, que trae como consecuencia un alto nivel de victimización y percepción de inseguridad.

La formulación del problema se planteó de la siguiente manera: Problema Principal: ¿Qué resultados se lograron en la ejecución del plan local de seguridad ciudadana, en un municipio limeño, en el periodo de 2017-2018? Los problemas específicos fueron: a) ¿Qué resultados se lograron en integridad en la ejecución del plan local de seguridad ciudadana, en un municipio limeño, en el periodo 2017-2018?, b), ¿ Qué resultados se lograron en multisectorialidad en la ejecución del plan local de seguridad ciudadana, en un municipio limeño, en el periodo 20172018?, c) ¿ Qué resultados se lograron en rigurosidad en la ejecución del plan local de seguridad ciudadana, en un municipio limeño, en el periodo 2017-2018? d) ¿Qué resultados se lograron en sostenibilidad en la ejecución del plan local de seguridad ciudadana, en un municipio limeño, en el periodo 2017-2018?, e)¿Qué resultados se lograron en intensidad en la ejecución del plan local de seguridad ciudadana, en un municipio limeño, en el periodo 2017-2018?.

Los objetivos de la investigación fueron: objetivo general: comprobar los resultados alcanzados en la ejecución del plan local de seguridad ciudadana, en un municipio limeño, en el periodo 2017-2018. Los objetivos específicos fueron: a) comprobar los resultados alcanzados en integridad en la ejecución del plan local de seguridad ciudadana, en un municipio limeño, en el periodo 2017-2018,b) comprobar los resultados alcanzados en multisectorialidad en la ejecución del plan local de seguridad ciudadana, en un municipio limeño, en el periodo 2017-2018, c) comprobar los resultados alcanzados en rigurosidad en la ejecución del plan local de seguridad ciudadana, en un municipio limeño, en el periodo 2017-2018, d) comprobar los resultados alcanzados en sostenibilidad en la ejecución del plan local de seguridad ciudadana, en un municipio limeño, en el periodo 2017-2018, y e) comprobar los resultados alcanzados en intensidad en la ejecución del plan local de seguridad ciudadana, en un municipio limeño, en el periodo 2017-2018.

\section{Marco Teórico}

Respecto a los antecedentes internacionales, según Granados (2018), compara y cuestiona el comportamiento de la asignación presupuestal respecto a la seguridad ciudadana, así como el nivel de eficiencia del gasto. La inseguridad y percepción de inseguridad, es también una preocupación de la sociedad, en el caso de Colombia se ha podido determinar que los mimos son fenómenos distintos o diferentes, aunque pareciera que debería haber una relación directa. Sostiene que la percepción de seguridad se ve influenciada por la valoración objetiva que hace la persona sobre el crimen, haciendo referencia a la inseguridad real, es decir la ocurrencia de los delitos que realmente ocurren en su barrio y una consideración subjetiva de éste que se origina en sus percepciones psicológicas, que muchas veces se ve influenciado por los diferentes medios de comunicación, creando sensacionalismo y originando representaciones imaginarias de determinados crímenes; Gelves ( 2019) expresa que la seguridad y la percepción de la inseguridad ciudadana son fenómenos diferentes; Rincón (2018) trae la relación de urbeviolencia y las políticas públicas, Focas ( 2018) indica que Latinoamérica, que solo representa el 8\% de la población mundial, sin embargo tiene el 33\% de los crímenes del mundo; Mugaah, and Aguirre (2018) manifiestan que las ciudades son los lugares donde se definen la política y la práctica de la seguridad ciudadana, y Graham (2017) establece que las estadísticas son 
indicadores del nivel de homicidios y que son aceptadas como seguridad y democracia. Estos problemas de la delincuencia son una gran preocupación de la ciudadana dado su incremento, comparables con las demás necesidades como son: el desempleo, la salud y la educación, entre otros.

Ramirez (2018) expresa que en estas épocas de neoliberalismo se plantean políticas de control sin rostro humano, Alda ( 2018) sostiene la posibilidad de que América Latina surja como actor relevantes en materia de seguridad ciudadana Bell (2019) indica que equívocamente se considera que un nivel más alto de inmigración se trasluce en un nivel más alto de crimen; Soria ( 2018) establece que si el gasto en seguridad pública se orientara a prevenir la inseguridad, gastos de ámbito social, infraestructura y que incidiera en el ámbito cultural, crecimiento y creación de empleo, se lograría una mayor igualdad y oportunidades. De la Puppaa and Sredanovicb (2017) consideran que los inmigrantes lo que buscan es la naturalización, tanto para protegerse de la policía y la deportación como mejores condiciones económicas por la crisis en sus países de origen. El éxodo de poblaciones africanas y árabes, en la parte de Europa y en América, la inmigración de Centro América hacia los Estados Unidos, crea resistencia de la población nativa porque consideran a la población inmigrante como la causante de la falta de empleo y el crecimiento de la delincuencia, aunque los inmigrantes busquen fundamentalmente naturalización, movilidad y seguridad. La ascensión de partidos políticos nacionalistas y en algunos países hasta "xenófonos", es de alguna manera respuesta a este sentir de una parte del electorado.

En Latinoamérica es imposible poder diseñar políticas regionales en materia de seguridad ciudadana, justamente por esta tendencia nacionalista que ha aparecido en algunos países de la región, perdiendo de esta manera la posibilidad de liderar en forma regional el combate a la criminalidad organizada.

Rodrigez ( 2018), Rodriguez (2018), Sanguino (2016), Sagastegui (2015), Viotti (2018) y Flom, and Post ( 2016); manifiestan que el problema de la inseguridad ciudadana también debería estudiarse no solamente tomando en cuenta al "delincuente", sino también al rol que cumplen los demás organismos del Estado en el combate a la delincuencia y que tengan la continuidad en el tiempo y cambios de gobierno; además respetando los derechos ciudadanos. Lo que ocurre con frecuencia es que en materia de orden público se dictan leyes más drásticas e incluso como ocurrió en el caso del Perú, se llevaron a acusar a las bandas criminales como terroristas y a juzgarlas en el fuero militar.

Gonzales (2016), Gomez ( 2015), Bustillo and Vellosos ( 2016), y Acuña y Chudnovsky (2017) manifiestan que la participación comunitaria y social en la consideración de la prevención del delito se ha hecho indispensable en toda Latinoamérica, por cuanto los gobiernos recurren con mayor frecuencia a la colaboración entre la policía y la comunidad como una forma de afrontar el incremento de la delincuencia y la inseguridad. De la misma manera se contempla el rol periférico que ha tenido Latinoamérica en la discusión mundial sobre el enfoque de la seguridad humana, por cuanto el paradigma de la seguridad ciudadana, que forma parte de la seguridad humana, ha prevalecido en la región. Es necesario resaltar que el concepto de seguridad ha llegado a tener nuevas dimensiones. 
Flom (2018), Bug \& Bukow ( 2017), Gonzales, Pardo and Izquierdo (2017), y Dietrich, Crabtree, and Verfasserln ( 2019), indican que uno de los grandes problemas en el tratamiento de la inseguridad ciudadana tiene que ver con la falta de coordinación entre los actores del Estado o de la coordinación en beneficio de sus propios intereses que el de la población. Tratar la seguridad ciudadana requiere tomar en cuenta los incentivos de todos los actores involucrados. En el caso de la Unión Europea, se recomienda analizar o estudiar las nuevas medidas de seguridad, combinado tres elementos principales: actitudes individuales (especialmente la confianza), contexto social y equilibrio costo/beneficio. El análisis determina que la confianza es esencial, pero de una manera mucho más compleja de lo que suele argumentar.

En cuanto a los antecedente nacionales, como lo sostiene: Gallardo (2018) indica que en la violencia uno de los factores que intervienen es la falta de un sistema adecuado para la supervisión de las armas letales y no letales, en ese sentido recomienda, tomando en cuenta los convenios nacionales e internaciones, de naturaleza vinculante y no vinculante; se debería concordar nuestras normas con los criterios de i) sistemas de licencias y autorizaciones, ii) marcaje, iii) tipificación penal y iv) registro; de la misma manera Mujica, Peñaloza, y Zevallos, desde el robo, acopio, acondicionamiento y disposición final.

Mendoza (2016), Malaver (2017), Montalvo (2018), Taboada (2016), y Patino (2016), manifiestan no obstante que en la última década se ha notado un mayor crecimiento de la economía, sin embargo, los índices de criminalidad se han incrementado notoriamente seguido por un aumento de la percepción de inseguridad ciudadana. Existen muchos factores para ello. Algunos son: falta de continuidad y coordinación entre las autoridades políticas locales y policiales, implementación de políticas públicas multicausales e intersectoriales y mejores presupuestos públicos. El gobierno ha buscado atender este problema de diferentes maneras, como el caso de la declaratoria de emergencia en el Gobierno Regional del Callao; Pero por su carácter transitorio mientras duró el estado de emergencia disminuyo la delincuencia, pero pasado el mismo volvieron los crímenes y violencia.

En cuanto a las teorías relacionadas con la investigación, tenemos la seguridad ciudadana según PNUD (2013a) es la sucesión de determinar, incrementar y preservar la condición civil y democrática, desterrando las conminaciones de violencia en los ciudadanos y facilitando su convivencia tranquila y en paz. A la seguridad ciudadana se le debe considerar como un bien público porque responde a la garantía eficaz de los derechos de las personas, esencialmente el derecho a la vida, a la probidad de las personas, a la inviolabilidad de los domicilios y la libertad de tránsito. De la misma manera indican que la seguridad ciudadana no solamente se refiere a la disminución de los delitos, sino a todo un conjunto de estrategias, de carácter exhaustivo y multifacética, en procura de acrecentar el nivel de vida de los ciudadanos; de acciones comunes para detectar a tiempo la criminalidad, el poder acceder a una adecuada justicia y recibir una educación fundada en valores con observancia a la ley y la consideración.

Chinchilla y Vorndrand ( 2018) sostienen que el nuevo paradigma de la Seguridad Ciudadana debe estudiarse considerando: a) la integridad, b) la multisectorialidad, c) rigurosidad, d) sostenibilidad y d) la escalabilidad. Donde la integridad está referida a las estrategias articuladas que impulsen la visión compartida de los problemas y los principios fundamentales, considerando las diferencias de los actores. La multisectorialidad, definida como las estructuras 
organizativas que faciliten la coordinación entre las autoridades de los diferentes estamentos de gobierno. La rigurosidad, que tiene que ver con la facilidad de la información y las estadísticas, el nivel de profesionalización de las personas y empleo de los adelantos tecnológicos en comunicación e información. La sostenibilidad y escalabilidad que contempla consideraciones como el liderazgo, fuentes de financiamiento permanentes y aspectos como el diseño y planificación.

Otras teorías relacionadas como la gestión pública, partiendo que es una responsabilidad del Estado a quien corresponde administrar, gestionar y controlar los asuntos públicos, y quien ejerce la autoridad sobre un territorio y sus habitantes; define las formas de organización burocrática en función del monopolio de la violencia legítima Acuña y CHudnovsky (2017). Estos autores hacen de igual manera una diferenciación entre administración pública y gestión pública, al sostener que la administración pública es la estructura administrativa del Estado. La administración pública lo definen en una forma simple como todos los procesos, organizaciones e individuos vinculados con el cumplimiento de los dispositivos legales y otras normas emitidas por las legislaturas, los tribunales y los gobiernos; mientras que la gestión pública son las formas de gestionarla. Sostienen de igual manera, que el proceso de la gestión pública ha pasado de una etapa donde predominó el paradigma burocrático Weberiano, que incidía en aspectos meritocráticos y racionales para buscar una administración pública de calidad a un nuevo paradigma a partir de la década de los noventa, que denominan "la segunda generación" que se caracteriza por la aparición de reformas pro mercado y disminución del aparato del Estado. Este nuevo paradigma denominado la Nueva Gestión Pública (NGP), trata de adecuar herramientas de la gestión de la administración privada a la gestión pública, buscando atender los requerimientos de los ciudadanos con enfoques de eficiencia, eficacia y competitividad.

Pliscoff (2017) expresa que la Nueva Gestión Pública se orienta hacia un Estado mucho más eficiente, más cercano a los deseos y anhelos de los ciudadanos, viéndolos como hace la gestión privada, como clientes que demandan una mejor atención. Esta nueva manera de comprender la gestión pública, se basa en tres pilares conceptuales: la primera que es la contractualización, definida como una herramienta de gestión orientada a proporcionar los servicios o tareas anteriormente desarrolladas por instituciones o servidores públicos. La segunda denominada mayor discreción de los funcionarios públicos, es decir dar mayor autonomía a los empleados públicos en sus actos de gestión y por último la tercera: búsqueda de resultados, usando por excelencia el presupuesto por resultados (PrR), como herramienta de gestión concediendo inclusive estímulos a aquellas entidades que logran alcanzar las metas y objetivos establecidos.

\section{Método}

El tipo de investigación fue participativa, estudio que emerge a partir de un problema que tiene su origen en la comunidad, y que busca la solución con la finalidad de mejorar la calidad de vida de las personas inmersas en el problema Caicedo y Mardones ( 1998). El enfoque de este estudio fue cualitativo, para Hernandez y Mendoza ( 2018), un estudio cualitativo busca estudiar un fenómeno de una forma sistemática. Pero, en vez de partir de una teoría y luego compararlo con el mundo empírico, el investigador parte de un hecho concreto o particular y revisa los 
trabajos previos, de manera simultánea, con la finalidad de formular una teoría que guarde coherencia con lo que está investigando u observando.

El diseño de la investigación fue un estudio de caso. Como sostiene Denny (1978, p.370), citado por Alonso (2006) un estudio de caso es un examen completo o intenso de una faceta, una pregunta o tal vez eventos que tienen lugar en un marco geográfico a lo largo del tiempo.

Stake (1999) sostiene que el estudio de casos, es el estudio de la singularidad como también de la diversidad de un caso en particular, con la finalidad de entender su desenvolvimiento en momentos importantes. El caso se considera como algo específico, complejo, pero en funcionamiento. El caso es un sistema unificado y total. No es indispensable que operen o se desenvuelvan a la perfección, sus objetivos incluso pueden parecer irracionales, sin embargo, es un sistema. Debido a eso a los programas como también a las personas se les considera como casos evidentes.

Las técnicas a empleadas fueron: a) análisis sistemático o análisis documental, definido como la recopilación de la información de los documentos, materiales y diferentes artefactos. Por lo general las diferentes personas, las organizaciones, comunidades, países elaboran o producen diversa información, que es una valiosa fuente para obtener los datos para realizar las investigaciones. Hernández, Fernandez y Baptista (2014); b) la entrevista, entendida como un encuentro para trocar información o conversar entre personas, donde el investigador será el entrevistador, el que hace las preguntas, la otra persona será el entrevistado, quien responde las preguntas formuladas por el entrevistador. Puede darse entre dos o más personas. Como sostiene Janesick (1998, citado por Hernández, Fernandez y Baptista ( 2014), mediante la entrevista, se hace preguntas y se obtiene respuestas, se consigue una correspondencia y la elaboración de significados con relación a un tema en particular.

Los instrumentos a usados en el proceso de investigación fueron las fichas de análisis documental, lista de cotejo y las guías de entrevistas semiestructuradas. El procedimiento determinado en el trabajo de investigación, es aquella definida por Hernández, Fernandez y Baptista (2014).

La triangulación es el método por excelencia que es usada en la investigación cualitativa y en particular, en el estudio de casos, para comprender y cruzar los datos obtenidos, en el sentido de comprobar lo que hemos observado y lo que estamos presentando poseen el mismo significado cuando lo ubicamos en otras circunstancias. La triangulación puede ser de fuentes, triangulación de investigador, triangulación de teoría, y triangulación metodológica. Stake (1999). La triangulación en esta investigación está dada en la comprobación hecha de las entrevistas al comisario de Condevilla, al especialista en seguridad ciudadana, al ex representante de los comités de seguridad ciudadana, y a la víctima de robos y asaltos. También se hizo triangulación al evaluar los planes de seguridad ciudadana, y las actas del comité de seguridad ciudadana de los años 2017-2018.

Las unidades de análisis fueron la gestión de las autoridades políticas y policiales, del distrito de San Martin de Porres de los años 2017-2018, y las víctimas de actos delincuenciales. Participaron en las entrevistas un Comisario del distrito, un experto en temas de seguridad 
ciudadana, un ex integrante del Comité de Seguridad ciudadana y un vecino del municipio víctima de un hecho delictivo. Las entrevistas siguieron la metodología de la saturación y su posterior codificación en hechos relevantes para su triangulación. No se identificó teorías emergentes. De la misma manera se analizaron todas las actas de las sesiones del Comité de Seguridad ciudadana del distrito de San Martín de Porres realizadas en forma mensual, de los años 2017 y 2018; para ser contrastadas con la realidad y las respuestas de los entrevistados.

\section{Resultados}

El objetivo general de la presente investigación fue "comprobar los resultados alcanzados en la ejecución del plan local de seguridad ciudadana, en un distrito limeño, en el periodo 2017 2018", de acuerdo a la lista de cotejo aplicada a las actas de las sesiones del Comité de Seguridad ciudadana de los años 2017-2018, de acuerdo a las categorías establecidas, se obtuvieron los siguientes resultados, que se presentan en la tabla 1:

Tabla 1.

Consolidado de la evaluación de los planes de seguridad ciudadana del distrito Limeño de los años 2017-2018

\begin{tabular}{llllll}
\hline Categoría & $\begin{array}{l}\text { Cantidad de } \\
\text { Indicadores }\end{array}$ & Si & No & $\begin{array}{l}\text { Nivel de Cumplimiento } \\
(\%)\end{array}$ & $\begin{array}{l}\text { Nivel de Cumplimiento } \\
\text { valorativo }\end{array}$ \\
\hline Integridad & 9 & 9 & 0 & 100 & Alto \\
Rigurosidad & 3 & 1 & 2 & 33.33 & Medio \\
Sostenibilidad & 5 & 2 & 3 & 40 & Medio \\
Intensidad & 3 & 1 & 2 & 33.33 & Medio \\
\hline Total & 41 & 24 & 17 & 58.54 & Medio \\
\hline
\end{tabular}

Fuente: elaboración propia

El resultado mostrado en la Tabla 1, indica que el nivel de cumplimiento de los planes de seguridad ciudadana en el distrito investigado, califica con un nivel de cumplimiento valorativo de medio, respecto a las categorías establecidas. La única categoría que alcanza la totalidad es el de integridad, calificando para un nivel de cumplimiento valorativo alto. La multisectorialidad califica para un nivel de cumplimiento medio; la sostenibilidad y rigurosidad e intensidad, califican para un nivel de cumplimiento valorativo medio. Esto es nivel de eficacia lograda por el Comité de Seguridad ciudadana de lo planificado entre los años 2017-2018 y lo alcanzado.

Respecto al cumplimiento de las actividades verificadas y establecidas en las actas de sesiones del Comité de Seguridad Ciudadana del distrito, de los periodos 2017-2018, el resultado obtenido se presenta la Tabla 2: 


\section{Tabla 2}

Resultados de la evaluación de las actividades establecidas en las actas del Comité de Seguridad Ciudadana del Distrito Limeño, periodo 2017-2018

\begin{tabular}{|c|c|c|c|c|c|c|c|}
\hline $\mathbf{N}^{\circ}$ & Actividad & 2017 & $\%$ & 2018 & $\%$ & Incremento/Decremento & $\begin{array}{l}\text { Nivel de } \\
\text { Cumplimiento } \\
\text { Valorativo }\end{array}$ \\
\hline 1 & Administrativas y Formales & 14 & 23.32 & 66 & 27.5 & 371.42 & Alto \\
\hline 2 & Pedidos y reclamos & 12 & 20 & 56 & 23.33 & 366.67 & Alto \\
\hline 3 & $\begin{array}{l}\text { Adquisición de alarmas cámaras } \\
\text { de video vigilancia }\end{array}$ & 4 & 6.67 & 1 & 0.41 & -75 & Bajo \\
\hline 4 & $\begin{array}{l}\text { Adquisición de vehículos para } \\
\text { patrullaje }\end{array}$ & 1 & 1.67 & 0 & 0 & -100 & Bajo \\
\hline 5 & Capacitación & & & 22 & 9.17 & & Bajo \\
\hline 6 & $\begin{array}{l}\text { Actividades de coordinación } \\
\text { interinstitucional }\end{array}$ & 16 & 26.67 & 32 & 13.33 & 100 & Bajo \\
\hline 7 & $\begin{array}{l}\text { Informes de cumplimiento y } \\
\text { control }\end{array}$ & 11 & 18.33 & 60 & 25 & 536.36 & Alto \\
\hline 8 & Actividades presupuestales & 1 & 1.67 & 3 & 1.26 & 200 & Bajo \\
\hline \multirow[t]{2}{*}{9} & Construcción de más comisarías & 1 & 1.67 & 0 & 0 & -100 & Bajo \\
\hline & Total actividades & 60 & 100 & 240 & 100 & 300 & Alto \\
\hline
\end{tabular}

Fuente: elaboración propia

Como se aprecia en la tabla 2 hubo un desarrollo significativo en varias de las actividades impulsadas por el distrito a través del Codisec, en temas de seguridad ciudadana; pero de actividades administrativas y formales tuvieron un incremento de $371.42 \%$ respecto al año 2017; informes de cumplimiento y control, con un incremento del 536.36\%, el más alto de todos, de 11 informes realizados el año 2017 se pasó a 60 el año 2018; las actividades de índole presupuestal se incrementaron en $200 \%$ y las actividades de coordinación interinstitucional en $100 \%$. Las actividades de pedidos y reclamos también se incrementaron significativamente, de 12 pedidos y reclamos del año 2017 pasaron a 56 el año 2018, lo que representa un incremento del $366.67 \%$. Por otro lado vemos que en temas específicos de seguridad ciudadana y cuyo cumplimiento sí incide directamente en la seguridad ciudadana, no se ejecutaron o tuvieron un decrecimiento; tal es el caso de la adquisición de alarmas y cámaras de video de vigilancia, que de 4 adquiridas en el 2017, solo se adquirió 1 en el 2018; lo que representa un decremento del $75 \%$, igual pasó en la adquisición de vehículos de patrullaje, se adquirió 1 en el año 2017 y ninguno el año 2018; decremento del $-100 \%$ y finalmente la construcción de comisarias, de una construida el año 2017, se pasó ninguna el año 2018; decremento del -100\%. La interpretación de esta tabla es que mientras el Comité de Seguridad ciudadana se preocupó por el desarrollo de actividades burocráticas o de coordinación interinstitucional; los reclamos de los ciudadanos no fueron atendidos oportunamente y menos aún desarrollaron actividades directamente en este sentido.

De acuerdo a los objetivos establecidos y las categorías determinadas; el análisis realizado en las entrevistas, se arriba a los siguientes resultados: respecto al primer objetivo específico que fue definido como "comprobar los resultados alcanzados en integridad en la 
ejecución del plan local de seguridad ciudadana, en un municipio limeño, en el periodo 20172018, se pudo determinar que en el caso del distrito de san Martín de Porres, existen manifestaciones que distan de lo recomendado como políticas adecuadas. Se han encontrado respuestas, entre otras, que el distrito tiene limitaciones o carencias respecto a las instituciones conformantes del Comité de Seguridad ciudadana (Codisec) por cuanto muchas de estas instituciones no asisten a las reuniones programadas, lo que indica una falta de compromiso y responsabilidad de los representantes de las instituciones; carencia de una visión integral de la seguridad ciudadana, cada institución elabora su propio plan de seguridad sin la participación de los ciudadanos; existe un trabajo desarticulado, alto índice de inseguridad en la zona, robos y asaltos que afectan a las personas y los negocios, muchas veces con consecuencias fatales; los planes de seguridad solo se formulan, pero no se cumplen, donde comisarías, serenazgo, gobiernos locales, congreso y alcaldes, según los ciudadanos, son los responsables de la inseguridad ciudadana en el distrito; sin embargo en la tabla 1, de acuerdo a la evaluación realizada entre lo planeado y ejecutado, de 9 indicadores para la municipalidad se lograron al $100 \%$. Si los entrevistados manifiestan que existen en este aspecto deficiencias, quiere decir que los mismos no están llegando a los ciudadanos.

El segundo objetivo específico se definió como "comprobar los resultados alcanzados en multisectorialidad en la ejecución del plan local de seguridad ciudadana, en un distrito limeño, en el periodo 2017-2018". Las respuestas obtenidas de parte de los entrevistados indican que en el distrito analizado existen carencias o deficiencias de índole logístico, falta de efectivos policiales, incumplimiento del patrullaje conjunto con el serenazgo, falta de unidades vehiculares, falta de coordinación entre la policía y la municipalidad, control inadecuado, desconocimiento en cuanto a la organización y funcionamiento del distrito, falta de más comisarías y patrulleros, poco tiempo de permanencia del comisario en el distrito, requerimiento de mayores rondas policiales, inadecuada distribución de patrulleros, deficiencias en el ministerio público, expedientes de denuncias policiales mal elaboradas, la municipalidad solo acude a la comisaría en busca de información estadística, faltan cámaras de video vigilancia, deficiencias en la ejecución del presupuesto, no toman en cuenta al Codisec, la tasa del arbitrio del serenazgo no lo pagan los contribuyentes, el comisario no tiene el perfil comunitario, la municipalidad tiene problemas para ejecutar su presupuesto. Este indicador no fue considerado en la tabla 1; pero por los asuntos que ella contiene es posible compararlo con los resultados de la tabla 2, por estar vinculada a aspectos de infraestructura, coordinación y organización y en estos asuntos hubo incumplimientos de parte del Comité de Seguridad ciudadana del distrito de San Martín de Porres.

El tercer objetivo específico se formuló como "comprobar los resultados alcanzados en rigurosidad en la ejecución del plan local de seguridad ciudadana, en un distrito limeño, en el periodo 2017-2018”. Las respuestas obtenidas alcanzan, al igual que los otros objetivos, opiniones divididas. Estas son: la existencia de falta de metas y objetivos claros, falta de un buen sistema de comunicación entre el serenazgo, la policía y la ciudadanía; importancia de ejecutar y hacer cumplir la ley, la demora de la respuesta de la policía incrementa la desconfianza en esta, no dan importancia a la información estadística, la dificultad de poder identificar a los delincuentes, por cuanto estos se disfrazan y hacen reglaje antes de actuar. La tabla 1 indica un nivel de cumplimiento medio, lo que confirma lo manifestado por los entrevistados. 
El cuarto objetivo específico fue "comprobar los resultados obtenidos en sostenibilidad en la ejecución del plan local de seguridad ciudadana, en un distrito limeño, en el periodo 20172018", de acuerdo a las respuestas obtenidas expresan limitaciones, entre otras, que los serenos no tienen la autoridad suficiente para detener personas, solo pueden hacer arresto ciudadano; son pocos los serenos que existen actualmente lo que impide una mayor presencia en el distrito; no existe un sistema radial compartido entre el serenazgo y la Policía Nacional, falta de interés de parte del gobierno local y nacional, de apoyar la organización de la sociedad civil, el impedimento legal que los serenos puedan portar armas de fuego; poca asignación presupuestal para aspectos de seguridad nacional, repercusión de la delincuencia en la economía, problema agudizado por la inmigración, necesidad de la inmediatez, de parte de la policía y el serenazgo. La tabla 1 indica un nivel de cumplimiento medio, que queda confirmado con las entrevistas realizadas.

El último objetivo específico se planteó como "comprobar los resultados alcanzados en intensidad en la ejecución del plan local de seguridad ciudadana, en un distrito limeño, en el periodo 2017-2018”. Las respuestas obtenidas, fueron los siguientes: el serenazgo tiene poca presencia en el distrito, porque son muy pocos; lo ideal sería contar entre 500 a 700 serenos; igualmente se vuelve a reiterar el accionar lento de la policía, dudas del accionar de la Policía o del Ministerio Público, respecto a la detención del delincuente capturado, por cuanto posteriormente a este hecho se le ve caminar libremente; trabajo aislado de muchos sectores, por falta de una buena coordinación y hay un manifiesto incumplimiento de la ley de seguridad ciudadana. La tabla 1 indica un nivel de cumplimiento de 1, lo que queda confirmado con el resultado de las entrevistas realizadas.

\section{Discusión}

Esta investigación buscó comprobar los resultados logrados en la ejecución del plan de seguridad ciudadana del 2017-2018, en el distrito de San Martín de Porres, de acuerdo a la legislación vigente, en materia de seguridad ciudadana, instituciones especializadas en combatir al alto índice de delincuencia que afecta a todas ciudades del Perú, principalmente a la ciudad de Lima, inclusive mayores presupuestos para mejorar la infraestructura y personal dedicado a la lucha contra la delincuencia; ésta sin embargo, en vez de disminuir se ha incrementado.

En ese sentido, los trabajos previos realizados por Granados ( 2018), Gelves ( 2019), Rincón ( 2018), Focas (2018), Mugaah, And Aguirre (2018) y Graham (2017), quienes manifiestan "que el problema de la delincuencia es una preocupación de la ciudadanía, dado su incremento" (p.4), tema que coincide con lo encontrado en esta investigación, donde los entrevistados manifestaron que existe un "alto índice de inseguridad en la zona, robos y asaltos que afectan a los negocios". Estos mismos autores manifiestan que "el incremento de la violencia es fundamentalmente urbano y como esta puede ser abordada.... bajo los enfoques socioeconómicos espaciales, sociocultural, sociopolítico" (p. 5); que coincide con lo encontrado en esta investigación, por cuanto los entrevistados reconocen como elemento positivo lo realizado por la municipalidad en cuanto al "trabajo mancomunado con los grupos de interés, en búsqueda de la paz y la tranquilidad; sin embargo los resultados de la investigación, en materia de mayores asignaciones presupuestales, no coincide con lo expuesto por los investigadores, 
quienes manifiestan, que en el caso particular de Colombia, la "relación presupuesto y delitos no han sido inversamente proporcionales" (p.5).

Ramirez ( 2018), Alda (2018), Bell (2019), Soria (2018) y De la Puppaa And Sredanovicb ( 2017), expresan que "en los aspectos de la delincuencia y de la criminalidad también intervienen factores como la inmigración ilegal" (p.5), que coincide con lo encontrado en esta investigación, donde el entrevistado manifiesta "que los venezolanos son los principales autores de hechos delictivos".

Por otro lado, Rodrigez (2018), Rodriguez (2018), Sagastegui (2015), Flom (2018) y Flom and Post ( 2016), sostienen " que el problema de la inseguridad ciudadana también debería estudiarse no solamente tomando en cuenta al delincuente sino también al rol que cumplen los demás organismos del Estado en el combate a la delincuencia y que tengan continuidad en el tiempo y cambios de gobierno" (p.6), esto coincide con lo encontrado en la presente investigación, donde los entrevistados manifestaron " el accionar lento de la policía, dudas del accionar de la policía y del Ministerio Público, respecto a la liberación de delincuentes capturados, trabajo aislado de muchos sectores, falta de una buena coordinación y un manifiesto incumplimiento de la ley".

Otros autores internacionales referenciados son Gonzales (2016), Gomez ( 2015) y Bustillo, and Vellosos ( 2016), quienes manifiestan que "la participación comunitaria y social de la prevención del delito se ha hecho indispensable en toda Latinoamérica, por cuanto los gobiernos recurren con mayor frecuencia a la colaboración entre la policía y la comunidad como una forma afrontar el incremento de la delincuencia y la inseguridad" (p.7). Esta afirmación encuentra correlato en el resultado encontrado, porque se evidencia la forma como el gobierno local de San Martín de Porres, ha venido cumplimiento no solamente el mandato de la ley sobre la participación ciudadana, sino que se evidencia la participación de la ciudadanía en la seguridad ciudadana.

En cuanto a los autores Flom (2018), Gonzales, Pardo and Izquierdo ( 2017), y Dietrich, Crabtree, and Verfasserln (2019), quienes expresan que "los grandes problemas en la inseguridad tienen que ver con la falta de coordinación entre los actores del Estado en beneficio de sus propios intereses que el de la población. Tratar la seguridad ciudadana requiere tomar en cuenta los incentivos de los actores involucrados" (p. 7). Es posible que esta condición sea una de las principales causas del incremento de la delincuencia en el distrito, materia de investigación, por cuanto en el trabajo de investigación se ha encontrado manifestaciones claras de esta condición, es decir, " que las instituciones preparan sus propios planes de seguridad" , " el trabajo es desarticulado", " falta de apoyo de la municipalidad a las Comisarías" , "instituciones que no asisten a las reuniones del comité se seguridad ciudadana", " falta de compromiso de las instituciones y falta de responsabilidad", "planes de seguridad se formulan, pero no se cumplen".

Respecto a los antecedentes nacionales, encontramos a Gallardo( 2018), Brandt (2017), Mujica, Peñaloza y Zevallos, ( 2018), que indican "que en la violencia uno de los factores que intervienen es la falta de un sistema adecuado para la supervisión de las armas letales y no letales" (p. 7). En la investigación realizada se encontró manifiesta evidencia que los 
delincuentes actúan con armas de fuego y son muy peligrosos, lo cual indica que existen deficiencias en el uso y control de armas de fuego.

Otros de los autores nacionales, son Mendoza (2016), Malaver (2017), Montalvo(2018), Taboada (2016), y Patino (2016), manifiestan que "no obstante que en la última década se ha notado un mayor crecimiento de la economía, sin embargo, los índices de criminalidad se han incrementado notoriamente seguido por un aumento de la percepción de inseguridad ciudadana" (p.8). También precisan que existen muchos factores para ello, algunos son: "falta de continuidad y coordinación entre las autoridades políticas locales y policiales, implementación de políticas públicas multicausales e intersectoriales y mejor presupuestos públicos" (p.8). Este aporte realizado por estos investigadores queda confirmado con lo encontrado en la investigación realizada, por cuanto se ha manifestado que uno de los problemas que influye en la inseguridad ciudadana es la falta de continuación de las políticas de seguridad ciudadana y la falta de continuidad del comisario, además se requiere de un comisario que tenga la condición o el conocimiento comunitario y que no rote cada año.

Chinchilla y Vorndrand (2018), a quienes hemos tomado como referentes teóricos para categorizar esta investigación, indican que el "nuevo paradigma de la seguridad ciudadana debe estudiarse considerando la a) integridad, b) la multisectorialidad, c) rigurosidad, d) sostenibilidad y e) la escalabilidad", tal es así que la estructura de los cuestionarios básicos usados en las entrevistas, tomaron en cuenta esta categorización. Se evaluó también la elaboración y ejecución del plan de seguridad ciudadana, del distrito de San Martín de Porres, empleando esta misma categorización, cuyo resultado fue presentado en la tabla 1, lo que permitió determinar que el nivel de cumplimiento de los planes de seguridad ciudadana en el distrito de San Martín de Porres alcanzaron un nivel de cumplimiento valorativo de medio (58.54\%).

\section{Conclusiones}

Se ha comprobado el alto índice de criminalidad existente en el distrito de San Martín de Porres, no obstante que se ha venido aplicando la ley 27933 de seguridad ciudadana. La municipalidad de San Martín de Porres cumple con hacer los planes y formularlos de acuerdo a lo prescrito en la ley, pero en su aplicación y puesta en práctica encuentra diversas limitaciones, logrando un nivel de cumplimiento valorativo de medio (58.34\%.).

La evaluación de los planes de seguridad ciudadana del distrito de San Martín de Porres, del 2017-2018, se hicieron tomando en consideración la categorización recomendada por Chinchilla y Worndrand (2018), quienes consideran que la seguridad ciudadana debe estudiarse con los nuevos paradigmas de la integridad, la multisectorialidad; rigurosidad, sostenibilidad y escalabilidad (intensidad). Comparadas cada una de estas categorías se arribó a la conclusión que la mayoría de las mismas no con cumplidas a cabalidad.

El sistema de control, para verificar el cumplimiento del plan de seguridad ciudadana, no está claramente definida, debido a la superposición de sistemas de control, tanto de parte de la municipalidad, que tiene su propia Oficina de Control Interno (OCI), que ve el presupuesto público, y por otro lado los órganos de control del gobierno regional y el Ministerio del Interior, que están interesados por las estadísticas para saber si ha aumentado o disminuido el índice de 
victimización o percepción de inseguridad; que el cumplimiento de los programas de seguridad ciudadana.

Las autoridades y funcionarios integrantes del Comité de Seguridad Ciudadana (Codisec), no tienen el suficiente compromiso para hacer una lucha frontal contra la inseguridad, a esto hay que agregar la transitoriedad de los comisarios, quienes por lo general solamente permanecen un año en la comisaría del distrito, imposibilitando aplicar políticas de seguridad ciudadana en un mayor tiempo.

Distancia y falta de confianza de los ciudadanos en la Policía Nacional y el Serenazgo municipal por cuanto no se nota su presencia en el distrito, y no hay prontitud y capacidad de respuesta ante un pedido de ayuda.

\section{Bibliografía}

Ministerio del Interior . (2018). Diagnóstico de la Seguridad Ciudadana en el Perú 2013-2018. Dirección General de Información para la Seguridad: Viceministerio de Seguridad Pública , Lima , Lima.

PNUD_ Programa de la Naciones Unidad para el. (enero de 2013(a)). Sinopsis: seguridad ciudadana. Prevención de crísis y recuperación. Obtenido de www.undp.org/cpr

27933, L. N. (12 de Febrero de 2003). Ley que crea el Sistema Nacional de Seguridad y sus Reglamentos D.S.011-2014-IN y D.S.012-2003-IN. Lima, Perú: Diario Oficial el Peruano

Acuña, C. y CHudnovsky, M. (2017). 12 Notas de concepto para entender mejor el Estado, las politicas públicas y su gestión. (C. A. Fomento, Productor) Obtenido de www. gobernabilidadcaf-com

Alda, S. (Enero-Junio de 2018). Los desafíos de América Latina para proyectarse como actor regional en el ámbito de la seguridad internacional. Revista de Relaciones Internacionales, Estrategia y Seguridad, 13(1).

Alke, J. (. (Julio-Dciembre de 2018). The war on the poor. The producction of insecurity in Latín América. Zed Books. Revista de Ciencias Saocilaeds y Humanidades Noesis, 27.

Alonso, A. (2006). Estudio de Casos . La Habana, Cuba : Félix Varela .

BELL, B. (2019). Crime and Inmigration: Do poor labor market opportunities lead to migrant crime? Crime and immigration:IZA word of Labor 2019, 2(33).

Bug, M.\& Bukow, S.U. (2017). Civil Liberties Vs. Security. Why Citizens Accept o Rejerct Digital Security Measures. German Politics , 26(2), 292-313.

Bustillo, I. and Vellosos, H. (2016). Insecurity and Development in Latin America and the Caribbean. Institute For National Strategic Security National Defense University Stable: PRISM, 5(4), 48-67.

Caicedo y Mardones. (1998). Elaboración de tesis e informes técnico-profesionales. (S. J. Conosur, Ed.) España.

Chinchilla, L. y Vorndrand, D. (2018). Desafíos e innovación en gestión y políticas públicas en los últimos 10 años. División de Información para Servir al Ciudadano. Banco Interamericano de Desarrollo-BID. Documento para discusión $N^{\circ} I D B-D P-640,1-14$.

De la Puppaa F. And Sredanovicb D.Y. (2017). Citizen to stay or Citizen to go?: Naturalizatión, Security and Mobility of Migrants in Italy. Journal of Inmigrants\& Refugee Studies, 15(4), 366-383. 
Dietrich, N., Crabtree, CH., and Verfasserln. (2019). Domestic Demand for Human Rights free speech and the freedom -security Trade. The Pensylvania State University And University of Michigan , 63(2), 356-353.

Flom, H. (2018). The Political Economy of Citizen Security. A Conceptual framework. Obtenido de Colection Ftfontagro : https://cutt.ly/criybye

Flom, H. and Post, A. (Octubre de 2016). Blame Avoidance and Policy Stability in The developing Democracies: The Politics of Public Security in Buenos Aires. Ingenta Connect, 49(1), 23 46.

Focas, B. (Junio de 2018). Miedo al crimen, prevención del delito y narcotráfico: desafío para las politicas públicas de seguridad ciudadana en América Latina. Entrevista a Lucía Dammert. (S. E. FLACSO, Ed.) URVIO, Revista Latinoamericana de Estudios de Seguridad(22), 102-108.

Gallardo, M. (julio de 2018). Aproximaciones al control de armas pequeñas y ligeras: tareas pendientes en el campo de la implementación de instrumentos internacionales en el Perú. Revistas de Derecho Internacional y Relaciones Internacionales: Ius Inter Gentes , 1(1).

García, V. (Julio-Diciembre de 2018). Dimensiones Locales y de la seguridad y la cooperación transfronteriza en la Frontera Amazónica de Brasil, Colombia y Perú. Opera(23), 59-80.

Gelves, J. (enero-abril de 2019). ¿ Cuáles determinantes se relacionan con la percepción de inseguridad?. Un análisis estadístico de inseguridad y espacial para la ciudad de Bogotá. D.C. Revista de Criminología, 61(1), 69-84.

Gomez, O. (2015). Alternative views of security in Latin America: Towards a global contribution to human security. Berghanh New York-Oxford.

Gonzales, Y. (2016). Varieties of Participatory Security: Assessing Comunity Participation in Policing in Latin America . Public Administration And Development .

Gonzales. E.G., Pardo.M.E. and Izquierdo, J.M. (2017). New Approach to traning in citizen security from The social Link University-Community. (U. d. Oriente, Ed.) Maestro y Sociedad, 14(4), 572-580.

Graham, D. (February de 2017). Before the Body Count. Homicide Estatistics and everyday security in Latin America. Journal of Latin American Studies, 49, 29-54.

Granados, J. (2018). Seguridad Ciudadana en Bogotá:análisis del presupuesto y su eficacia en el gasto local 201-2016. Revista de Derecho Administrativo (20), 201-238.

Hernandez, R. y Mendoza, C.P. (2018). Metodología de la Investigación, Las rutas cuantitativas, cualitativas y mixtas. México: McGraw-Hill Interamericana Editores S.A. .

Hernández, R., Fernandez, C. y Baptista, P. (2014). Metodología de la Investigación (Sexta Edición ed.). McGraw-Hill/ Interamericana Editores S.A.de V.C.

Malaver, C. (2017). La declaratoria de emergencia y su impacto en la seguridad ciudadana, caso Región Callao periodo 2015-2016. Tesis para optar el grado académico de Magíster en Ciencia Politica y Gobierno con mención en Políticas Públicas y Gestión Pública. Escuela de Posgrado de la Pontificia Universidad Católica del Perú.

Mendoza, W. (2016). En busca de la seguridad: la implementación de la política pública de seguridad pública en Arequipa: caso distrito de Socabaya. Título para optar el grado académico de Magíster en Ciencia Política y Gobierno, con mensión en Políticas Públicas y Gestión Pública. Escuela de Posgrado de la Pontificia Universidad Católica del Perú.

Montalvo, L. (2018). Regulación Constitucional del arresto ciudadano en el contexto de la seguridad ciudadana y el respeto a las libertades ciudadanas. Tesis para optar el grado académico de Maestro en Derecho con mención en Constitucional y Gobernabilidad. 
Lambayeque, Chiclayo: Escuela de Posgrado de Derecho de la Universidad Pedro Ruiz Gallo.

Mugaah, R. And Aguirre, K. (abril de 2018). Citizen security in Latin América: The Hard Facts. (I. \&. 2017, Ed.) Igarapé Institute, Strategic Paper 33, 15(4).

Mujica, J., Peñaloza, A. y Zevallos, N. (mayo-agosto de 2018). Mercados legales de objetos robados: compraventa de computadoras portátiles robadas en mercados de Lima. Revista Crimen, 60(2), 89-105.

Patino, V. (2016). Patrullaje integrado de seguridad ciudadana: experiencias en el distrito de la Victoria. Tésis para optar el grado académico de Magister en Ciencia Política y Gobierno con mención en Políticas Públicas y Gestión Pública. Lima, Perú: Escuela de Posgrado de la Pontificia Universidad Católica del Perú.

Pliscoff, C. (Enero-Abril de 2017). Implementando la nueva gestión pública: problemas y desafíos a la ética pública.El caso Chileno. Revista de Ciencias Sociales de la Universidad Autónoma de la Ciudad dde México(73), 141-167.

PNUD. (2013a). Sinopsis: Seguridad Ciudadana. Prevención de crísis y recuperación. Obtenido de www.undp.org/epr

Ramirez, J. (Diciembre de 2018). De la era de la migración al siglo de la seguridad: surgimiento de políticas de control con rosto (in) humano. (R. Y. Ecuador, Ed.) URVIO(23), 10-258.

Rincón, A. (Junio de 2018). Abordajes teóricos sobre la relación entre la seguridad ciudadana y la violencia urbana en Colombia. Una lectura crítica. (F. S. Ecuador, Ed.) URVIO; Revista Latinomaricana de Estudios de Seguridad(22), 86-100.

Rodrigez, R. (2018). Evaluación de la Seguridad Ciudadana: las instituciones de prevención, control y justicia penal de la República de Panamá como caso de estudio . Tesis para optar el grado de Doctor. Madrid España : Facultad de Ciencias Politicas y Sociales de la Universidad Complutense de Madrid, España .

Rodriguez, C. (2018). Análisis de la implementación de la politica pública de seguridad ciudadana en Bogotá (1995-2015). Tesis para optar el grado de Doctor. Madrid, España: Facultad de Ciencias Sociales, Universidad Complutense de Madrid, España.

Sagastegui, F. (2015). La seguidad Nacional en el Estado Constitucional de Derecho. Tesis para optar el grado de Doctor. Madrid, España: Departamento de Derecho Público del Estado, Universidad Carlos III, Madrid, España.

Sanguino, A. (2016). Elementos para una política de seguridad urbana. Tesis para optar el grado de Doctor. Madrid, España: Facultad de Ciencias Políticas y Sociales de la Universidad Complutense de Madrid, España.

Soria, R. (2018). Una estimación del costo de la inseguridad y de la delincuencia en México: análisis comparativo a nivel de las entidades Federativa. Revista de Gestión y Política Pública, XXVII(1), 111-147.

Soto, J. (. (2018). El Impacto del Terrorismo Islamista del siglo XXI en la Doctrina Estratégica de los Estados Unidos y la Federación Rusa: un estudio comparativo. (A. N. Estratégicos, Ed.) Política y Estrategia $N^{\circ} 132,47-94$.

Stake, R. (1999). Investigaciones con Estudio de Casos (Segunda Edición ed.). Madrid : Ediciones Morata S.L.

Taboada, N. (2016). Analisis de la política de seguridad ciudadana a partir del estudio de caso del Progama de Jóvenes Líderes entre los años 2008-2014. Tesis para optar el grado académico de Magíster en Ciencia Politica y Gobierno con mención en Políticas Públicas y Gestión Pública. Escuela de Posgrado de la Pontificia Universidad Católica del Perú.

Esta obra se comparte bajo la licencia Creative Common Atribución-No Comercial 4.0 International (CC BY-NC 4.0) 
Viotti, M. (2018). Sendero Punitivo de las pandillas en el Perú . Tesis para optar el grado de Doctor. Madrid, España : Facultad de ciencias Políticas y Sociología, Universidad Complutense de Madrid, España . 